Research Article

\title{
Assessment of new strategies in the management of guillain barre syndrome,cairo university
}

\begin{abstract}
Background, Guillain Barre Syndrome (GBS) deserves a serious attention in children. The treatment of GBS consists of both supportive and immune-modulator treatments, among which intravenous immunoglobulin (IVIG) and plasma exchange (PE) are considered most effective. We aimed to assess the outcome of new strategies in the management of moderate to severe cases.
\end{abstract}

Methodology, This is a retrospective study including 38 GBS children, divided into 3 groups. Group I (30 children) received concomitant therapy of intravenous steroid with IVIG, group II ( 5 children) received 2 courses, 2 weeks apart, of IVIG ( $0.4 \mathrm{~g} / \mathrm{kg} /$ day) over 5 days each, and group III ( 3 children) received 2 courses of PE, 5 sessions each, 2 weeks apart.

Results, Death occurred only among cases of group I (10/20 cases) $33.3 \%$. Children in group I showed regressive course $(p=0.01)$. Higher incidence of mechanical ventilation was found in group II (66.6\%). Cases in group II stayed longer (median=77days) than those in group I or III.

Conclusion, The combination of IVIG and intravenous steroid can be used to hasten recovery in moderate GBS. The use of 10 sessions of PE can decrease the need for mechanical ventilation. Bulbar affection upon admission can be considered as a sign of severity.

Keywords: acute demyelinating polyradiculopathy, intravenous immunoglobulin, plasmapheresis, steroids
Volume I Issue 4 - 2014

Hanaa I Rady,HamsAttala

Department of Pediatrics, Cairo University, Egypt

\begin{abstract}
Correspondence: Hanaa I Rady, Department of Pediatrics, Faculty of Medicine, Cairo University, 5 Gameat El doual El arabia Street, Mohandesseen, Cairo I24I I, Egypt, Tel 201000000000,Email hannaarady@gmail.com
\end{abstract}

Received: September 02, 2014 | Published: September 12 , 2014
Abbrevations: GBS,guillain barre syndrome; PE,plasma exchange; IVIG,intravenous immunoglobulin; MFS, miller fisher syndrome; CSF,cerebrospinal fluid; PICU,pediatric intensive care unit; UL,upper limbs; LL,lower limbs; IVMP,intravenous methylprednisolone; EMG,electromyography; $\mathrm{NCV}$,nerve conduction velocity

\section{Introduction}

Guillain Barre Syndrome (GBS) is an inflammatory polyneuropathy characterized by acute onset, rapid progressive, ascending symmetric muscular weakness, pain, and paresthesia. The progressive phase peaks in 7 to 14 days and can lead to various levels of weakness, from abnormal gait to total paralysis, cranial nerve weakness, pain, respiratory compromise, and autonomic instability. ${ }^{1}$ The autonomic manifestations include fluctuations in the heart rate, blood pressure, vasomotor stability, sweating and continence. ${ }^{2}$ The incidence of GBS in the pediatric age group is 0.8 cases per 100.000 . The prevalence of which depends on the geographic region.

The etio-pathogenesis of GBS has been hypothesized to involve a direct immune mediated mechanism against the peripheral nerves components, including the myelin sheath and the axon. ${ }^{1,3}$ Fifty to seventy percent of the cases are preceded by respiratory or gastrointestinal infectious episodes either bacterial or viral, less likely by vaccination. The strongest relationship is with infection by Camplylobacterjejuni ${ }^{1}$ and Mycoplasma pneumoniae. ${ }^{4}$

GBS is heterogeneous disease with various subtypes. Recognition of these subtypes is of clinical importance since each subtype has an independent pathogenesis and different type of pathology and prognosis. The subtypes are, acute inflammatory demyelination polyradiculoneuropathy, acute motor axonal neuropathy, and unclassified. ${ }^{5}$ Miller Fisher syndrome (MFS) is a clinical variant of GBS, characterized by acute onset ophthalmoplegia, ataxia, and areflexia. ${ }^{6}$ The diagnosis of GBS is made on clinical criteria, albuminocytologicaldissociation in cerebrospinal fluid (CSF) and the nerve conduction studies. Although GBS is a serious disease, recovery is the rule in children with very low morbidity and mortality. ${ }^{7}$ It is always complete, without any significant difference in the duration of recovery. ${ }^{8}$ Poor prognostic factors are related to the presence of severe disability on admission, cranial nerve affection, the need for mechanical ventilator and electrophysiological types (axonal form). ${ }^{9,10}$

The treatment of GBS consists of both supportive and immunomodulatory treatments, among which intravenous immunoglobulin (IVIG) and plasma exchange (PE) are considered most effective. ${ }^{11}$ The majority of children achieving a complete functional recovery within 6 months from the onset of illness. ${ }^{12}$ Therapeutic IVIG is capable of neutralizing neuromuscular blocking antibodies in GBS by dose dependent, antibody mediated mechanism. ${ }^{13}$ Therapeutic PE is an extracorporeal blood purification technique designed for the removal of large molecules weight substances from plasma. $^{14}$

PE is superior to IVIG regarding the duration of mechanical ventilation, with a tendency for a shorter pediatric intensive care unit (PICU) stay but not the short term neurological outcome. ${ }^{15}$ Although not clinically established, there may be a role for the concomitant use of steroids with IVIG ${ }^{16}$ and, in patients with severe disease and poor prognostic scores, PE followed by IVIG may be beneficial. ${ }^{17}$

More randomized trials comparing IVIG with PE concluded that giving IVIG after PE did not confer significant extra benefit. ${ }^{18}$ Under 
investigation are new treatment strategies, based on prognostic factors and more specific immune-modulations. ${ }^{19}$ Trials of interferon beta1a, brain-derived neurotrophic factor and CSF filtration, showed no significant benefit or harm. Another trial showed that the Chinese herbal medicine tripterygiumpolyglycoside hastened recovery significantly more than corticosteroids but this result needs confirmation. ${ }^{20}$

We aimed to compare the outcome of different treatment protocols applied for resistant cases of moderate and severe GBS and to highlight the idea that extending the treatment duration (example, 10 sessions of PE instead of 5) for resistant cases should be tried.

\section{Patients and methods}

This retrospective study was done over a period of 2 years (June 2010 till June 2013), in PICU of AbolReish EI Monira Hospital, Kasr Al Ainy Cairo University. The capacity of this PICU is 7 beds.

We included all cases of moderate and severe GBS admitted to this PICU in the study period who didn't improve after the standard treatment. Patients' age ranged from 3 to 11 years old. We excluded cases of mild GBS, chronic cases and cases referred to this PICU with previous treatment trials.

\section{We defined}

i. Mild GBS, patients with involvement of upper limbs (UL) and lower limbs (LL) only and over a period of $>48 \mathrm{hrs}$. They received IVIG.

ii. Moderate GBS, patients with involvement of UL and LL over a period of $<48 \mathrm{hrs}$ with no bulbar or autonomic manifestation, or involvement of UL \& LL over a period of $>48 \mathrm{hrs}$ with bulbar or autonomic manifestation) and they received concomitant therapy of IVIG with methylprednisolone. IVIG therapy was given at a dose of $400 \mathrm{mg} / \mathrm{kg} /$ day for 5 days. Steroid in a form of intravenous methylprednisolone (IVMP) for 3 days ( $1^{\text {st }}$ day, $30 \mathrm{mg} / \mathrm{Kg}$, $2^{\text {nd }}$ day, $20 \mathrm{mg} / \mathrm{kg}, 3^{\text {rd }}$ day, $10 \mathrm{mg} / \mathrm{Kg}$ ), followed by oral prednisone at a dose of $2 \mathrm{mg} / \mathrm{Kg}$ for one week then withdrawal by decreasing $5 \mathrm{mg}$ every 2days.

iii. Severe GBS, patients with respiratory muscles involvement at presentation or UL \& LL over $<48$ hrs with autonomic manifestation and/or bulbar involvement). Standard treatment of these patients was 5 secessions of PE every other day with regular monitoring to blood pressure, electrolyte [21]. Non-improving cases were treated with either one of those protocols, IVIG and PE 2 weeks apart, 10 sessions of PE 2 weeks apart or 2 courses of IVIG ( $2 \mathrm{~g} / \mathrm{Kg}$ each $) 2$ weeks apart.

In the plasma exchange we used an intermittent flow machine with filtration technique. The type of replacement fluids were mainly albumin and in few cases fresh frozen plasma due to financial issues concerning availability of albumin.

Clinical data was recorded on a standardized sheet, which includes, patient's age, sex, date of admission and discharge, antecedent events, the order of neurological affection UL, LL, trunk, bulbar, respiratory muscles) progression of involvement over a period of (less than $48 \mathrm{hrs}$ or more than $48 \mathrm{hrs}$ ) and associated symptoms (autonomic manifestation or sensory loss), course (regressive, progressive or stationary). The hospital course was analyzed including feeding (intravenous fluids, ryle or oral feeding), need for oxygen support or mechanical ventilator requirement, electromyography (EMG) and nerve conduction velocity (NCV), outcome, function status of patients. All cases were diagnosed by the clinical picture upon presentation and by EMG and NCV.
We compared and analyzed all the data and outcome of cases with moderate (30 patients) and severe ( 8 patients) GBS.

\section{Statistical Data}

Nominal data were expressed as frequency and percentage and were compared using Chi square test. Numerical data were expressed as mean, standard deviation and range and compared using $\mathrm{T}$ test, that t-tests were used to compare the moderate and severe GBS groups. Non-parametric data were compared using Mann Whitney test. Pearson's correlations were used to explore associations between numerical variables. A $p$ value $<0.05$ was considered statistically significant.

\section{Results}

A retrospective study was done on 2 groups of GBS patients (moderate and severe cases) using different treatment protocols (38 patients), admitted to PICU, in Children hospital of Cairo University. We compared different unusual lines of treatment (three treatment protocols used in this PICU). i. Group I included 30 GBS patients received concomitant therapy of intravenous steroid with IVIG. ii. Group II included 5 GBS patients received IVIG over 5 days $(400 \mathrm{mg} / \mathrm{kg} /$ day) then another 5 days IVIG 2 weeks later when no improvement was observed.

iii. Group III included 3 GBS patients received 10 secessions of $\mathrm{PE}$ in the form of 5 secessions every other day, another 5 secessions 2 weeks later when no improvement was observed.

Treatment groups had no significant complications attributable to treatment intervention apart from minor hypotension episodes.

Table 1, described the data of the studied cases with their characteristics, lines of treatment and outcome. Although the duration of hospital stay was much longer in cases receiving the 2 courses of IVIG (77 days) compared with others yet, this difference was statistically insignificant (Table 2). Mortality rate was $26.3 \%(10$ cases from 38 cases). All deaths (10 cases) were in Group I (33.3\%), however it was not statistically significant (Table 3).

Table I Analysis of data of the studied cases

\begin{tabular}{|c|c|c|}
\hline \multicolumn{2}{|c|}{ Number of Cases (Percentage) } & \\
\hline \multicolumn{3}{|l|}{ Sex } \\
\hline \multirow{2}{*}{\multicolumn{2}{|c|}{ - $\quad$ Female }} & $15(39.47)$ \\
\hline & & $23(60.53)$ \\
\hline$\bullet$ & Male & \\
\hline \multicolumn{3}{|c|}{ Duration of Hospital Study } \\
\hline$\bullet$ & 2 wks & $20(52.63)$ \\
\hline$\bullet$ & More than 2 wks & $18(47.37)$ \\
\hline \multicolumn{3}{|l|}{ Season } \\
\hline$\bullet$ & Winter & $7(18.42)$ \\
\hline$\bullet$ & Spring & $13(31.58)$ \\
\hline • & Autumn & $5(13.16)$ \\
\hline & & $14(36.84)$ \\
\hline$\bullet$ & Summer & \\
\hline
\end{tabular}


Table Continued...

Number of Cases (Percentage)
Proceeding Symptoms
- URTI
- GE
- Viral infection
- Post-vaccination
- None

Progression of Weakness Over

- $\quad>48 \mathrm{hrs}$

- $\quad<48$ hrs

Autonomic Symptoms

- No

- Yes

Bulbar Symptoms

- Yes

- No

Respiratory Muscles Affection

- Yes

- No

Feeding

- Oral

- $\quad$ Ryle

- Intravenous fluids

\section{Course}

- Stationary

- $\quad$ Progressive

- Regressive

Mechanical Ventilation

- $\quad$ Yes

- No

Outcome

- Died

- Discharged

28 (73.68)

Treatment

- IVIG + steroids

- IVIG 2

- $\quad \mathrm{PE}>5$ secessions

URTI, upper respiratory tract infection; GE, gastroenteritis; viral infection seasonal influenza virus, cytomegalovirus, epstein barr virus; IVIG, intravenous immunoglobulin; IVIG 2, 2 courses of IVIG; PE, plasma exchange

Table 2 Duration of hospital stay in relation to treatment protocols.

\begin{tabular}{lllll}
\hline & Group I & Group II & Group III & P-value \\
$\begin{array}{l}\text { Duration of hospital } \\
\text { stay (Median in Days) }\end{array}$ & 9.5 & 77 & II & 0.88 \\
\hline
\end{tabular}

*P value is non-significant

Table 3 Outcome in relation to treatment protocols

\begin{tabular}{lllll}
\hline Outcome & $\begin{array}{l}\text { Group I } \\
\mathbf{n}=\mathbf{3 0}\end{array}$ & $\begin{array}{l}\text { Group II } \\
\mathbf{n = 5}\end{array}$ & $\begin{array}{l}\text { Group III } \\
\mathbf{n = 3}\end{array}$ & $\boldsymbol{P}$ - value \\
Died/discharged & $10 / 20$ & $0 / 5$ & $0 / 3$ & 0.05 \\
\hline
\end{tabular}

*P value is non-significant

Table 4 Need of mechanical ventilation in relation to treatment protocols.

\begin{tabular}{lllll} 
Mechanical & Group I & Group II & Group III & P-value \\
Ventilation & $\mathbf{n = 3 0}$ & $\mathbf{n = 5}$ & $\mathbf{n}=\mathbf{3}$ & \\
Yes/No & $13 / 17$ & $2 / 3$ & $\mathrm{I} / 2$ & 0.5 \\
\hline
\end{tabular}

$* P$ value is non-significant

The percent of mechanical ventilation was higher in Group II $(66.6 \%)$ when compared with Group I and Group III $(43.3 \%$ and $33.3 \%$, respectively), yet it was not statistically significant (Table 4) When comparing the course of illness with the different treatment protocols we found that Group I cases (IVIG and steroid) had more regressive course than the others $(p=0.01)$.

We also compared different signs on presentation (bulbar, automatic and respiratory muscles affection) with (length of hospitalization, mechanical ventilation and outcome)

i. Bulbar affection was more associated with death outcome $p=0.001$.

ii. Discharged cases had higher association with autonomic manifestations $p=0.001$.

iii. Autonomic manifestations was associated with more regressive course $p=0.03$ and the need for mechanical ventilation $p=0.01$.

iv. Respiratory muscles affection was associated with the need of mechanical ventilation $p=0.01$.

GBS deserves serious attention and a study like this in children is especially valuable since almost all previous studies were done entirely in adults.

Our three treatment groups had no significant complications attributable to treatment intervention apart from minor hypotension episodes responding to fluid boluses, this confirms the safety of these therapeutic regimens.

In the El Bayoumi et al. ${ }^{15}$ no serious adverse events were encountered. However, in five trials for which information was available for the Cochrane review, there were more adverse events in the PE than the IVIG group. ${ }^{22}$

Our total mortality rate $26.3 \%$ is considered high when compared with that reported by Pritchard ${ }^{23}(10 \%)$ and by Xiao et al. ${ }^{24}(5 \%)$.

Although death was reported only with cases in group I (defined as moderate GBS), our study reported that there is no significant association between treatment modality and outcome and death is mostly related to other PICU issues related to infections as pneumonia. This agreed with results of El Bayoumi et al. ${ }^{15}$ as PE and IVIG had equivalent efficacy in terms of improvement. We reported no deaths in cases treated with PE $(0 / 5)$, while in the review done by the Cochrane Collaboration, ${ }^{25}$ they reported $4.6 \%$ death in cases treated with PE $(15 / 321)$. 
Our study showed no statistical significant difference between lines of treatment and length of hospitalization.

Hospital stay in group I (9.5 days) was shorter than group II (77 days) and group III (11 days), but with no statistical significance. That is why we explained the variation in length of stay by the occurrence of ventilator associated pneumonia that complicated cases on mechanical ventilator.

El Bayoumi et al. ${ }^{15}$ showed that PE group had a tendency for a shorter PICU stay, but wasn't statistically significant $(p=0.094)$. And although steroids were considered ineffective in treatment of GBS, ${ }^{23}$ we observed that when intravenous methylprednisolone is combined with IVIG it hasten recovery. In a case report, it was found that it decrease the radicular pain associated with GBS. ${ }^{26}$

In our study no statistically significant association was found between MV and lines of treatment, but mechanically ventilated cases were more in group II (66.6\%) than group I (43.3\%) and III (33.3\%).

No significant difference in efficacy of IVIG and PE for duration of requirement for artificial ventilation was found in two large trials in adults. ${ }^{27}$

On the other hand, El Bayoumi et al. ${ }^{15}$ study in children, reported that the duration of MV was significantly shorter in the PE group than IVIG group.

We found significant association between autonomic manifestation and the need for MV and also, significant association between respiratory muscle affection and the need for MV. And these were normal expected findings.

We observed strong association between autonomic manifestations and the regressive course of the disease with more tendencies to be discharged from PICU, but no explanation was found to this observation.

\section{Conclusion}

We concluded that combination of IVIG with IVMP can be used to hasten recovery in moderate GBS. The use of 10 sessions of PE can decrease the need for MV and hence its complications. We considered bulbar affection upon admission as sign of severity.

\section{Limitations}

Our study main limitation was the discrepancy between the number of cases in each of the compared groups and this was out of hands. Further studies on larger scales is recommended.

\section{Acknowledgments}

None.

\section{Conflicts of Interest}

There is no conflict of interest.

\section{Funding}

None.

\section{References}

1. Rosen BA. Guillain-Barre syndrome. Pediatr Rev. 2012;33(4):164-170.

2. Dimario FJ, Edwards C. Autonomic dysfunction in childhood GuillainBarre syndrome. J Child Neurol. 2012;27(5):581-586.
3. Pavone P, Pratico AD, Ruggieri M, et al. Acquired peripheral neuropathy: a report on 20 children. Int J Immunopathol Pharmacol. 2012;25(2):513-517.

4. Sharma MB, Chaudhry R, Tabassum I, et al. The presence of mycoplasma pneumonia infection and GM1 ganglioside antibodies in Guillain-Barre syndrome. J Infect Dev Ctries. 2011;5(6):459-464.

5. Alexander M, Prabhakar AT, Aaron S, et al. Utility of neurophysiological criteria in Guillain-Barre' syndrome: subtype spectrum from a tertiary referral hospital in India. Neurol India. 2011;59(5):722-726.

6. Lee KY. Anti-GQ1b-negative Miller Fisher syndrome after Campylobacter jejuni enteritis. Pediatr Neurol. 2012;47(3):213215 .

7. Koul RL, Alfutaisi A. Prospective study of children with Guillain-Barre syndrome. Indian J Pediatr. 2008;75(8):787-790.

8. Kannan MA, Ch RK, Jabeen SA, et al. Clinical, electrophysiological subtypes and antiganglioside antibodies in childhood Guillain-Barre syndrome. Neurol India. 2011;59(5):727-732.

9. Incecik F, Ozlem Herguner M, Altunbasak S. Guillain-Barre syndrome in children. Neurol Sci. 2011;32(3):381-385.

10. Halawa EF, Ahmed D, Nada MA. Guillain-Barre syndrome as a prominent cause of childhood acute flaccid paralysis in post polio eradication era in Egypt. Eur J Pediatr Neurol. 2011;15(3):241-246.

11. Harel M, Shoenfeld Y. Intravenous immunoglobulin and Guillain-Barre syndrome. Clin Rev Allergy Immunol. 2005;29(3): 281-287.

12. Sladky JT. Guillain-Barre syndrome in children. J Child Neurol. 2004;19(3):191-200.

13. Buchwald $\mathrm{B}$, Ahangari $\mathrm{R}$, Weishaupt $\mathrm{A}$, et al. Intravenous immunoglobulins neutralize blocking antibodies in Guillain-Barre syndrome. Ann Neurol. 2002;51(6):673-680.

14. Jayasena YA, Mudalige SP, Manchanayake GS, et al. Physiological changes during and outcome following 'filtration' based continuous plasma exchange in Guillain-Barre Syndrome. Transfusion \& Apher Sci. 2010;42(2):109-113.

15. El-Bayoumi MA, El-Refaey AM, Abdelkader AM, et al. Comparison of intravenous immunoglobulin and plasma exchange in treatment of mechanically ventilated children with Guillain-Barre syndrome: a randomized study. Critical Care. 2011;15(4):R164.

16. Shahar E, Leiderman M. Outcome of severe Guillain-Barre syndrome in children: comparision between untreated cases versus gamma-globulin therapy. Clinical Neuropharmacol. 2003;26(2):84-87.

17. Shahrizaila N, Yuki N. The role of immunotherapy in Guillain-Barre syndrome: understanding the mechanism of action. Expert Opinion Pharmacother. 2011;12(10):1551-1560.

18. Hughes RAC, Swan AV, van Doorn PA. Intravenous immunoglobulin for Guillain-Barre syndrome. Cochrane Database of Systematic Reviews. 2010;(6):CD002063.

19. Walgaard C, Jacobs BC, van Doorn PA. Emerging drugs for GuillainBarre syndrome. Expert Opin Emerg Drugs. 2011;16(1): 105-120.

20. Hughes RA, Pritchard J, Hadden RD. Pharmacological treatment other than corticosteroids, intravenous immunoglobulin and plasma exchange for Guillain-Barre syndrome. Cochrane Database Syst Rev. 2011;(3):CD008630.

21. Raphael JC, Chevret S, Jars-Guincestre MC, et al. Treatment of Guillain-Barre syndrome by plasma exchange: proposal of a therapeutic strategy. Rev Neurol. 1996;152(5):359-364.

22. Hughes RA, Swan AV, van Doorn PA. Intravenous immunoglobulin for Guillain-Barre syndrome. Cochrane Database Syst Rev. 2010;7:CD002063. 
23. Pritchard J. Novel therapeutic approaches to Guillain-Barre syndrome Expert Opin Investig Drugs. 2000;9(10):2307-2318.

24. Xiao J, Simard AR, Shi FD, et al. New Strategies in the Management of Guillain-Barre Syndrome. Clin Rev Allergy Immunol (in press). 2013.

25. Raphael JC, Chevret S, Hughes RAC, et al. Plasma exchange for Guillain-Barre syndrome. Cochrane review, prepared and maintained by The Cochrane Collaboration and published in the Cochrane Library. Issue 2 by John Wiley \& Sons, Ltd. 2008.
26. Endo K, Yasui K, Hasegawa Y, et al. An adult Guillain-Barre syndrome patient with enhancement of anterior roots on spinal MRI and severe radicular pain relieved by intravenous methylprednisolone pulse therapy: A case report. Rinsho Shinkeigaku. 2013;53(7):543-550.

27. Plasma Exchange/Sandoglobulin Guillain-Barre Trial Group. Randomised trial of plasma exchange, intravenous immunoglobulin, and combined treatments in Guillain-Barre syndrome. Lancet. 1997;349(9047):225-230. 